immune-tolerance by permitting effector cells to perpetrate hepatocyte damage.

Competing interests None declared.

\section{PMO-138 THE MOLECULAR MECHANISMS OF B CELL AND B CELL LYMPHOMA RECRUITMENT TO THE HUMAN LIVER}

doi:10.1136/gutjnl-2012-302514b.138

\begin{abstract}
${ }^{1,2} \mathrm{~S}$ Shetty, ${ }^{* 1} \mathrm{~T}$ Bruns, ${ }^{1} \mathrm{C}$ Weston, ${ }^{1} \mathrm{Z}$ Stamataki, ${ }^{1,2} \mathrm{Y}$ 0o, ${ }^{3} \mathrm{~S}$ Hubscher, ${ }^{4} \mathrm{~S}$ Jalkanen, ${ }^{1} \mathrm{P}$ Lalor, ${ }^{1,2} \mathrm{D}$ H Adams. ${ }^{1}$ Centre for Liver Research, University of Birmingham, UK; ${ }^{2}$ NIHR Liver Biomedical Research Unit, Birmingham, UK; ${ }^{3}$ Department of Pathology, Queen Elizabeth Hospital, Birmingham, UK; ${ }^{4}$ Meditcity Research, University of Turku, Turku, Finland
\end{abstract}

Introduction There is gathering interest in the presence of B cells within liver tissue and their contribution to chronic inflammation and fibrosis but the recruitment signals for B cells into peripheral tissue is poorly understood. In addition a large proportion of lymphomas which infiltrate the liver are of B cell origin but again little is understood of the mechanism that underlies this process. Lymphocyte recruitment to the liver occurs within the hepatic sinusoidal channels. These low shear vascular beds are lined by specialised hepatic sinusoidal endothelial cells (HSEC). Our aim was to understand the molecular mechanisms of $\mathrm{B}$ cell and $\mathrm{B}$ cell lymphoma recruitment to the liver.

Methods We used isolated human HSEC in flow assays with purified peripheral blood B cells to elucidate the molecular mechanisms of $B$ cell recruitment via HSEC. The contribution of conventional adhesion molecules, ICAM-1 and VCAM-1 and unconventional molecules VAP-1 and CLEVER-1/stabilin-1 was assessed by using function blocking antibodies. We repeated our experiments with two B cell lymphoma cell lines, CRL-2261 and Karpas B cell line. We assessed the contribution of chemokines by performing transwell assays and adding chemokines to our flow assays. We also tracked the motility of B cells and lymphoma cell lines on HSEC using tracking software. Results B cells were captured from flow and firmly adhered to HSEC, the primary adhesion receptor on HSEC was VCAM-1. B cells also underwent transendothelial migration which was mediated by a combination of ICAM-1, VAP-1 and CLEVER-1/stabilin-1. Lymphoma cell line recruitment shared several features of primary lymphocyte homing, firm adhesion was mediated by ICAM-1 and VCAM-1 and they demonstrated shape-change and crawling behaviour which was ICAM-1 dependent. The lymphoma cell lines did not undergo transendothelial migration and this could not be initiated with the addition of SDF-1 $\alpha$

Conclusion There is increasing evidence that B cells play an important role in chronic inflammatory liver diseases. The recruitment signals we have identified for B cells in this study may provide potential therapeutic targets for liver disease. Furthermore we have demonstrated preserved lymphocyte homing mechanisms in malignantly transformed B cells. These properties could be therapeutic targets to prevent lymphoma dissemination to the liver.

Competing interests None declared.

\section{PMO-139 HUMAN CYTOMEGALOVIRUS INFECTION OF HUMAN HEPATIC SINUSOIDAL ENDOTHELIAL CELLS PROMOTES CD4 T CELL ADHESION AND TRANSMIGRATION}

doi:10.1136/gutjnl-2012-302514b.139

${ }^{1} \mathrm{~T}$ Bruns, ${ }^{*}$ A Pachnio, ${ }^{1} \mathrm{P}$ F Lalor, ${ }^{2} \mathrm{P}$ A Moss, ${ }^{1} \mathrm{D}$ H Adams. ${ }^{1}$ Centre for Liver Research and NIHR Biomedical Research Unit in Liver Disease, Birmingham, UK; ${ }^{2}$ School of Cancer Sciences, University of Birmingham, Birmingham, UK

Introduction Animal studies suggest that sinusoidal endothelial cells and not hepatocytes are the site of cytomegalovirus (CMV) latency and reactivation in the liver and the source of secondary viral spread. Furthermore, murine CMV infection of sinusoidal endothelium is able to break immunotolerance and induce a strong $\mathrm{T}$ cell effector response. The aim of this study was to investigate, whether CMV infection of human hepatic sinusoidal endothelial cells (HSEC) modulates the ability of the liver to recruit and activate lymphocyte. Methods Recombinant endotheliotropic eGFP-labelled CMV was propagated in RPE-1 cells and purified by ultracentrifugation in tartrate/glycerol gradients. Primary HSEC were isolated from explanted livers, grown to confluence and infected with CMV over $2 \mathrm{~h}$. Infection was confirmed by fluorescence microscopy and plaque assay on fibroblasts. Chemokines and adhesion molecules were quantified by ELISA. Isolated primary lymphocytes and CMVspecific CD4 T cell clones were perfused over HSEC monolayers under constant flow simulating physiological shear stress and adhesion and transmigration recorded using phase contrast microscopy. Trans-well assays were used to study the phenotype of transmigrated cells using flow cytometry.

Results Human sinusoidal endothelial cells were permissive to CMV infection. CMV infection induced secretion of CXCL10 and CCL5 as well as an up-regulation of VCAM-1 and ICAM-1 surface expression. Early CMV infection resulted in a fourfold increase in the adhesion of allogeneic lymphocytes to infected HSEC monolayers compared with mock-infected endothelium. Under flow, transendothelial migration of CMV-reactive CD4 T cell clones was increased through CMV-infected endothelium and could be significantly reduced by the use of anti-CXCL10 antibodies. Transmigrated allogeneic CD4 CD45RO+ T cells and CMV-reactive T cell clones displayed increased expression of the early activation marker CD69 after transendothelial migration through CMV-infected HSEC.

Conclusion CMV infection of HSEC facilitates the up-regulation of cell-adhesion molecules and chemokines resulting in increased adhesion, transmigration and activation of CD4 $\mathrm{T}$ cells. This may explain how human CMV infection not only provokes significant hepatitis but also increases hepatic immune activation in graft rejection.

Competing interests None declared.

\section{PMO-140 ANALYSIS OF EUS-GUIDED CYST ASPIRATE HAS NO IMPACT ON SURGICAL MANAGEMENT OF SUSPECTED PANCREATIC CYSTIC TUMOUR}

doi:10.1136/gutjnl-2012-302514b.140

V Mahesh, ${ }^{*}$ W Tam, 0 N Nguyen. Department of Gastroenterology, Royal Adelaide hospital, Adelaide, Australia

Introduction Preferred strategies for evaluation and management of patients with pancreatic cysts remain controversial. EUS-guided fine needle aspiration (FNA) of suspected pancreatic cyst tumours for CEA and cytology is often recommended to evaluate malignant potential in order to guide further management.

Aim To evaluate the clinical impact of EUS guided cyst aspirate on surgical management of patients with suspected pancreatic cystic tumours.

Methods Outcome data of all patients having undergone EUS guided FNA of suspected pancreatic cystic tumours from March 2004 to November 2011 were retrospectively reviewed. Data were collected on demographics, EUS findings, radiological findings, biochemical and cytological findings, clinical outcomes and management. The mean follow-up was 24.5 months.

Results Of 123 patients (74F:49M; 64 \pm 2.7 years) with suspected pancreatic cystic tumours, only 10 (8\%) patients had surgical resection for IPMN with low grade dysplasia $(n=7), M C N(n=1)$, pancreatic neuroendocrine tumour $(\mathrm{n}=1)$ and serous cystadenoma $(\mathrm{n}=1)$. Only $3(12 \%)$ patients with CEA $>192 \mu \mathrm{g} / \mathrm{l}$ and $1(7 \%)$ with 